Pacific Journal of Mathematic 


\title{
CAPACITY DIFFERENTIALS ON OPEN RIEMANN SURFACES
}

\author{
Georges G. Weill
}

1. Introduction. We study in this report some orthogonal decompositions of the space $\Gamma_{h}$ of harmonic differentials of finite norm, on a Riemann surface $W$. We obtain generalizations of the known decompositions (I)

$$
\begin{aligned}
& \Gamma_{h}=\Gamma_{h m}+\Gamma_{h s e}^{*} \\
& \Gamma_{h}=\Gamma_{h 0}+\Gamma_{h e}^{*} .
\end{aligned}
$$

We then prove some existence theorems for differentials on $W$ harmonic except for the singularity $d z /(z-\zeta)$, of finite norm on $W-\Delta$, where $\Delta$ is a disk about $z=\zeta$.

A necessary and sufficient condition for their existence is the existence on $W-\Delta$ of a differential in $\Gamma_{h}(W-\delta)$ with nonzero period about the boundary $\beta$ of $W$.

We then construct "Green's differential”, "Capacity differentials", and prove some of their properties on compact bordered Riemann surfaces. The orthogonal property of Green's differential is extended to open hyperbolic Riemann surfaces.

\section{Some subspaces of $\Gamma_{h}$.}

2A. Let $\bar{W}$ be a compact bordered Riemann surface, with boundary $\beta$. Partition $\beta$ into $\gamma$ and $\delta=\beta-\gamma$ where $\gamma$ is a union of contours $\gamma_{i}$. We shall define the following subspaces of $\Gamma_{h}$ :

$$
\begin{aligned}
\Gamma_{h(0 \gamma)} & =\left\{\omega: \omega \in \Gamma_{h}, \omega=0 \text { on } \gamma\right\} . \\
\Gamma_{h(s e \gamma)} & =\left\{\omega: \omega \in \Gamma_{h}, \int_{\gamma_{i}} \omega=0\right\} .
\end{aligned}
$$

Those subspaces are clearly closed. We shall denote by $\Gamma_{h(m \gamma)}$ the subspace $\Gamma_{h e} \cap \Gamma_{h(0 \gamma)}$. We shall prove some orthogonal decomposition theorems.

THEOREM.

$$
\Gamma_{h}=\Gamma_{h(m \gamma)}+\Gamma_{h(s e \gamma)}^{*} \cap \Gamma_{h(o \delta)}^{*} .
$$

Proof. Let $\omega \in \Gamma_{h}^{\prime}$ and $d f^{*} \in \Gamma_{h(m \gamma)}^{*}$. Then $\left(\omega, d f^{*}\right)=\int_{\beta} \omega \bar{f}=$ $\sum_{i} \bar{f}_{\gamma_{i}} \int_{\gamma_{i}} \omega+\int_{\delta} \omega \bar{f}$ where $\bar{f}_{\gamma_{i}}$ is the constant value of $\bar{f}$ on $\gamma_{i}$. Now, if

Received August 21, 1961. 
$\omega \in \Gamma_{h(s e \gamma)} \cap \Gamma_{h(o \delta)}, \int_{\gamma_{i}} \omega=0$, and $\int_{\delta} \omega \bar{f}=0$. It follows that $\left(\omega, d f^{*}\right)=0$. Conversely, if $\left(\omega, d f^{*}\right)=0$, then $\sum_{i} \bar{f}_{\gamma_{i}} \int_{\gamma_{i}} \omega+\int_{\delta} \omega \bar{f}=0$.

Select $f=1$ on one of the $\gamma_{i}$, say $\gamma_{i_{0}}, f=0$ an $\delta$ and all other $\gamma_{i}$.

It follows that $\int_{\gamma_{i_{0}}} \omega=0$. This is true for any contour $\gamma_{i_{0}}$. Hence $\omega \in \Gamma_{h(s e \gamma)}$. Now take $f=0$ on $\gamma$; then $\int_{\delta} \omega \bar{f}=0$ for all such $f$. This readily implies $\omega=0$ on $\delta$, which proves the theorem.

2B, Define $\hat{W}_{\gamma}$ to be the double of $\bar{W}$ with respect to $\gamma$. It is obtained by partial welding of $\bar{W}$ along $\gamma$. It can be shown by a method analogous to the one in (I. Chapter V. \$14) that the harmonic differentials which can be continued to $\hat{W}_{\gamma}$ form the subspace $\Gamma_{h(0 \gamma)}+\Gamma_{h(0 \gamma)}^{* 1}$.

2C. We shall consider here the subspace:

$$
\Gamma_{h e(0 \delta)}=\left\{\omega: \omega \in \Gamma_{h}, \omega=d f, f=0 \text { on } \delta\right\} .
$$

The following theorem gives an orthogonal decomposition of $\Gamma_{h}$ involving $\Gamma_{h(s e \gamma)}^{*}$ :

$$
\text { Theorem. } \quad \Gamma_{h}=\Gamma_{h(s e \gamma)}^{*}+\Gamma_{h e(0 \delta)} \cap \Gamma_{h(0 \gamma)} \text {. }
$$

Proof. Let $d f^{*} \in \Gamma_{h(0 \gamma)} \cap \Gamma_{h e(0 \delta)}, \omega \in \Gamma_{h} . \quad$ Then $(\omega, d f)=\int_{\beta} \omega \bar{f}=$ $\Sigma \bar{f}_{\gamma_{i}} \int_{\gamma_{i}} \omega+\int_{\delta} \omega \bar{f}=\Sigma \bar{f}_{\gamma_{i}} \int_{\gamma_{i}} \omega$. If $\omega \in \Gamma_{h(s e \gamma)}$, then $\int_{\gamma_{i}} \omega=0$, and $\left(\omega, d f^{*}\right)=$ 0 . Conversely if $\left(\omega, d f^{*}\right)=0$, then $\Sigma \bar{f}_{\gamma_{i}} \int_{\gamma_{i}} \omega+\int_{\delta}^{\gamma_{i}} \omega \bar{f}=0$. Take $f=1$ on $\gamma_{i_{0}}, f=0$ elsewhere. Then $\int_{\gamma_{i_{0}}} \omega=0$ for any $\gamma_{i_{0}}$ and $\omega \in \Gamma_{h(\text { ser })}$.

2D. The next theorem gives an orthogonal decomposition of $\Gamma_{h}$, involving $\Gamma_{h(0 \gamma)}$.

THEOREM.

$$
\Gamma_{h}=\Gamma_{h(0 \gamma)}+\Gamma_{h e(0 \delta)}^{*} .
$$

Proof. Let $d f^{*} \in \Gamma_{h e(0 \delta)}^{*}, \omega \in \Gamma_{h}$. Then $\left(\omega, d f^{*}\right)=\int_{\beta} \omega \bar{f}=\int_{\gamma} \omega \bar{f} . \quad$ If $\omega \in \Gamma_{h(0 \gamma)}, \int_{\gamma} \omega f=0=\left(\omega, d f^{*}\right)$. Conversely, if $\left(\omega, d f^{*}\right)=0$, then $\int_{\gamma} \omega \bar{f}=$ 0 . This readily implies $\omega=0$ on $\gamma$, hence $\omega \in \Gamma_{h(0 \gamma)}$.

2E. We shall now extend our results to open Riemann surfaces. Let $W$ be an open Riemann surface. Consider a closed partition of the ideal boundary $\beta$ into $\gamma$ and $\delta=\beta-\gamma$. Consider a neighborhood of $\delta$, say $N_{0}(\delta)$, bounded by a set of contours $\delta_{0}$. $\delta_{0}$ divides $W$ into $N_{0}(\delta)$ 
and $W-N_{0}(\delta)$. We shall exhaust $W_{0}=W-N_{0}(\delta)$, using a regular exhaustion $\left\{\Omega_{n}\right\}$. Let $\omega_{h(0 \gamma)} \in \Gamma_{h(0 \gamma)}$. The restriction of $\omega_{h(0 \gamma)}$ to $\Omega$ has a decomposition:

$$
\left.\omega_{h(0 \gamma)}\right|_{\Omega}=\omega_{h(0 \gamma) \Omega}+\omega_{h e\left(0 \delta_{0}\right) \Omega}^{*} .
$$

Where $\omega_{h(0 \gamma) \Omega} \in I_{h(0 \gamma)}(\Omega)$ and $\omega_{h e\left(0 \delta_{0}\right) \Omega} \in \Gamma_{h e\left(0 \delta_{0}\right)}(\Omega)$. If $\Omega^{\prime} \supset \Omega, \omega_{h(0 \gamma) \Omega}-\omega_{h(0 \gamma) \Omega}-$ $\omega_{h(0 \gamma) \Omega^{\prime}}=\omega_{h e\left(0 \delta_{0}\right) \Omega^{\prime}}^{*}-\omega_{h e\left(0 \delta_{0}\right) \Omega}^{*}$ where the right hand side is an element of $\Gamma_{h e\left(0 \delta_{0}\right)}(\Omega)$ and therefore is orthogonal to $\omega_{h(0 \gamma) \Omega}$ on $\Omega$. It follows that

$$
\left\|\omega_{h(0 \gamma) \Omega}-\omega_{h(0 \gamma) \Omega^{\prime}}\right\|_{\Omega}^{2}=\left\|\omega_{h(0 \gamma) \Omega^{\prime}}\right\|_{\Omega}^{2}-\left\|\omega_{h(0 \gamma) \Omega^{\prime}}\right\|_{\Omega}^{2} .
$$

Therefore $\left\|\omega_{h(0 \gamma) \Omega}\right\|_{\Omega}$ increases with $\Omega$. But it is also bounded, for the orthogonal decomposition $\left.\omega_{h(0 \gamma)}\right|_{\Omega}=\omega_{h(0 \gamma) \Omega}+\omega_{h e\left(0 \delta_{0}\right) \Omega}^{*}$ shows that $\left\|\omega_{h(0 \gamma) \Omega}\right\|_{\Omega} \leqq\left\|\omega_{h(0 \gamma)}\right\|_{\Omega} \leqq\left\|\omega_{h(0 \gamma)}\right\|$. We find that $\left\|\omega_{h(0 \gamma) \Omega}\right\|_{\Omega}$ has a finite limit and this implies that

$$
\left\|\omega_{h(0 \gamma) \Omega}-\omega_{h(0 \gamma) \Omega^{\prime}}\right\|_{\Omega} \rightarrow 0 \text { as } \Omega \text { and } \Omega^{\prime} \rightarrow W_{0} .
$$

For a fixed $\Omega_{0}$, the triangle inequality gives: $\left\|\omega_{h(0 \gamma) \Omega^{\prime}}-\omega_{h(0 \gamma) \Omega^{\prime \prime}}\right\|_{\Omega_{0}} \rightarrow 0$ as $\Omega^{\prime}, \Omega^{\prime \prime} \rightarrow W_{0}$ independently of each other. We conclude (I. Chapter II. Theorem $13 \mathrm{C})$ that $\omega_{h(0 \gamma) \Omega}$ tends to a harmonic limit differential $\omega_{h(0 \gamma) W_{0}}$. Furthermore:

$$
\left\|\omega_{h(0 \gamma) \Omega}-\omega_{h(0 \gamma) W_{0}}\right\|_{\Omega} \rightarrow O \text { as } \Omega \rightarrow W_{0} .
$$

Let now $\sigma^{*} \in \Gamma_{h e\left(0 \delta_{0}\right)}^{*}$. Then $\left(\omega_{h(0 \gamma) W_{0}}, \sigma^{*}\right)_{\Omega}=\left(\omega_{h(0 \gamma) W_{0}}-\omega_{h(0 \gamma) \Omega}, \sigma^{*}\right)$; as $\Omega \rightarrow$ $W_{0}$. Then for $\delta_{\nu} \subset \Omega$

or

$$
\left(\omega, \sigma^{*}\right)=\lim _{\nu \rightarrow \infty}\left(\omega, \sigma_{\nu}^{*}\right)=\lim _{\nu \rightarrow \infty}\left[\lim _{\Omega \rightarrow W}\left(\omega-\omega_{h(0 \gamma) \Omega}, \sigma_{\nu}^{*}\right)_{\Omega}\right]
$$

$$
\begin{array}{r}
\left|\left(\omega, \sigma^{*}\right)\right|^{2} \leqq \lim _{\nu \rightarrow \infty}\left[\lim _{\Omega \rightarrow W}\left\|\omega-\omega_{h(0 \gamma) \Omega}\right\|_{\Omega}^{2}\right] . \\
\left\|\sigma_{\nu}^{*}\right\|_{\Omega}^{2} \leqq \lim _{\nu \rightarrow \infty}\left[\lim _{\Omega \rightarrow W}\left\|\omega-\omega_{h(0 \gamma) \Omega}\right\|_{\Omega}^{2}\left\|\sigma_{\nu}^{*}\right\|_{W}^{2}\right] \\
=\lim _{\Omega \rightarrow W}\left\|\omega-\omega_{h(0 \gamma) \Omega}\right\|_{\Omega}^{2} \cdot \lim _{\nu \rightarrow \infty}\left\|\sigma_{\nu}^{*}\right\|_{W}^{2} .
\end{array}
$$

The last limit being finite, it follows that $\left(\omega, \sigma^{*}\right)=0$. We conclude that $\omega \in \Gamma_{h(0))}(W)$. Thus $\Gamma_{h(0 \delta)}(W)$ is formed precisely by those differentials which can be approximated by differential of class $\Gamma_{h(0))}(\Omega)$.

We state this result as a theorem.

Theorem. $\Gamma_{h(0 \gamma)}(W)$ is the limit of $\Gamma_{h(0 \gamma)}(\Omega)$ for $\Omega \rightarrow W$ in the sense that $\omega \in \Gamma_{h(0 \gamma)}(W) \Longleftrightarrow$ there exists differentials $\omega_{h(0 \gamma) \Omega} \in \Gamma_{h(0 \gamma)}(\Omega)$ such that $\left\|\omega-\omega_{h(0 \gamma) \Omega}\right\|_{\Omega} \rightarrow 0$. 
2F. We shall now extend Theorem $2 \mathrm{C}$ to open surfaces.

THEOREM. On an arbitrary Riemann surface

$$
\Gamma_{h}=\Gamma_{h(s e \gamma)}^{*}+\Gamma_{h(0 \gamma)} \cap \Gamma_{h e(0 \delta)} .
$$

Proof. It is easy to see that $\Gamma_{h(s e \gamma)} \perp \Gamma_{h(0 \gamma)}^{*} \cap \Gamma_{h e(0 \delta)}^{*}$. Let $\sigma \in \Gamma_{h(s e \gamma)}$ and $\omega \in \Gamma_{h(0 \gamma)} \cap \Gamma_{h e(0 \delta)}$. Consider a canonical exhaustion $\{\Omega\}$. Let $\omega$ be approximated in norm by $\omega_{\Omega} \in \Gamma_{h(0 \gamma)}(\Omega) \cap \Gamma_{h e(0 \delta)}(\Omega)$. Then, $\Omega$ being canonical, $\left(\sigma, \omega_{\Omega}^{*}\right)_{\Omega}=0$ thus $\left(\sigma, \omega^{*}\right)_{\Omega}=\left(\sigma, \omega^{*}-\omega_{\Omega}^{*}\right)$ and the inner product can be made arbitrarily small, while $\Omega$ is arbitrarily large. Hence $\left(\sigma, \omega^{*}\right)=0$ and the orthogonality is proved.

Conversely, if $\omega \in \Gamma_{h}$ and $\omega \perp \Gamma_{h(s e r)}^{*}(W)$, for a canonical $\Omega$ let $\omega_{1 \Omega}$ be the projection of $\omega$, restricted to $\Omega$ on $\Gamma_{h(0 \gamma)} \cap \Gamma_{h e(0 \delta)}$. Then $\omega-$ $\omega_{1 \Omega} \in \Gamma_{h(s e \gamma)}^{*}(\Omega)$. For $\Omega^{\prime} \supset \Omega$, we conclude that $\omega_{1 \Omega}-\omega_{1 \Omega^{\prime}} \in \Gamma_{h(s e \gamma)}^{*}(\Omega)$, hence $\omega_{1 \Omega}-\omega_{1 \Omega^{\prime}} \perp \omega_{1 \Omega}$. Therefore $\left\|\omega_{1 \Omega}-\omega_{1 \Omega^{\prime}}\right\|_{\Omega}^{2}=\left\|\omega_{1 \Omega^{\prime}}\right\|_{\Omega}^{2}-\left\|\omega_{1 \Omega}\right\|_{\Omega}^{2} \leqq\left\|\omega_{1 \Omega^{\prime}}\right\|_{\Omega^{\prime}}^{2}-$ $\left\|\omega_{1 \Omega}\right\|_{\Omega}^{2}$. It follows that $\left\|\omega_{1 \Omega}\right\|_{\Omega}^{2}$ increases with $\Omega$. But $\left\|\omega_{1 \Omega}\right\| \leqq\|\omega\|$. Therefore $\omega_{1}=\lim _{\Omega \rightarrow W} \omega_{1 \Omega}$ exists and lies in $\Gamma_{h(0 \gamma)} \cap \Gamma_{h e(0 \delta)}$. Furthermore because $\omega-\omega_{1 \Omega} \in \Gamma_{h(s e \gamma)}(\Omega)$ and every dividing cycle lies in an $\Omega$, it follows that $\omega-\omega_{1} \in \Gamma_{h(s e \gamma)}^{*}\left(W_{1}\right)$. On the other hand, $\omega \perp \Gamma_{h(s e \gamma)}^{*}$ by assumption and $\omega \perp \Gamma_{h(s e \gamma)}^{*}$. We conclude that $\omega-\omega_{1}$ and $\Gamma_{h(0 \gamma)} \cap$ $\Gamma_{h e(0 \delta)} \perp \Gamma_{h(s e \gamma)}^{*} . \quad \Gamma_{h(s e \gamma)}$ being closed, $\Gamma_{h(s e \gamma)}^{*}$ is $\perp \Gamma_{h(0 \delta)} \cap \Gamma_{h e(0 \delta)}$.

\section{Existence theorem.}

3A. We shall now prove some existence theorems for harmonic differentials with a singularity of the type $d z /(z-\zeta)$. Let $W$ be an open Riemann surface, $z=\zeta$ a point of $W$. Let us consider a disk $\Delta$ mapped on $|z|<1$ such that $\zeta \in \Delta$. Select $r_{1}$ and $r_{2}$ positive such that $|\zeta|<r_{1}<r_{2}<1$. Construct a function $e_{1}(z) \in C^{2}$ which has value 1 for $|z|<r_{1}$ and value 0 for $|z|>r_{2}$, and the function $e_{2}(z)$ such that $e_{1}+$ $e_{2}=1$ on $W$.

Let $\underline{W}=W-\left\{z:|z|<r_{1}\right\}$. We shall call $\alpha_{0}$ the contour $|z|=r_{1}$. Let us assume that on $\underline{W}$ there exists a reproducing differential for $\alpha_{0}$, say $\sigma\left(\alpha_{0}\right)$. To $\sigma\left(\alpha_{0}\right)$ corresponds an analytic differential on $\underline{W}: \omega=\sigma\left(\alpha_{0}\right)+$ $i \sigma^{*}\left(\alpha_{0}\right)$. Denoting by $q$ the period of $\omega$ around $\alpha_{0}$, we consider $\varphi=$ $(2 \pi i / q) \omega$. In the annulus $r_{1}<|z|<r_{2}, d z /(z-\zeta)-\varphi$ is exact; let $\Phi$ be an analytic function such that $d \Phi=d z /(z-\zeta)-\varphi$ in the annulus. Notice that $\Phi$ is defined up to an additive constant. We now construct the following differential:

$$
\Theta=e_{1} d z /(z-\zeta)+\Phi d e_{1}+e_{2} \varphi
$$

$\Theta$ is an element of $C^{1}$ and is closed on $W$ punctured at $z=\zeta$. Moreover $\Theta-i \Theta^{*}=0$ near the singularity and in a boundary neighborhood. Hence 
$\Theta$ is square integrable and by de Rham's decomposition theorem:

$$
\Theta-i \Theta^{*}=\omega_{e 0}+\omega_{h}+\omega_{e 0}^{*} .
$$

Then $\tau=\Theta-\omega_{e 0}=i \Theta^{*}+\omega_{b}+\omega_{e 0}^{*}$ is closed and coclosed in any region which does not contain $z=\zeta$. $\tau$ is therefore harmonic on $W$ except for the singularity $d z /(z-\xi)$. Such a differential is necessarily unique; in fact, let $\tau$ and $\tau^{\prime}$ be 2 solutions corresponding to the same $\Theta$. Then $\tau-\tau^{\prime}$ is harmonic and $\tau-\tau^{\prime} \in \Gamma_{e 0}$. Therefore $\tau-\tau^{\prime}=0$. We shall remark that two different functions $\Phi$, differing by a constant $C$ will yield the same $\tau$ : for in $\Theta, C d e$, is an element of $\Gamma_{e 0}$, hence immaterial for the definition of $\tau$.

3B. Let us consider a closed partition of the ideal boundary $\beta$ of $W$ into 2 parts $\gamma$ and $\delta$, and the corresponding partition into $\gamma^{\prime}=\alpha_{0} \cup \gamma$ and $\delta$ for $\underline{W}$. On $W$ we perform the decomposition:

$$
\omega_{h}=\omega_{1}^{*}+\omega_{2}
$$

where $\omega_{1}^{*}=\Gamma_{h(s e s)}^{*}(W)$ and $\omega_{2} \in \Gamma_{h e(0 \gamma)}(W) \cap \Gamma_{h(08)}(W)$. Then $\tau=i\left(e_{1} d z /(z-\right.$ $\left.\zeta)+\Phi d e_{1}\right)^{*}+e_{2} \varphi+\omega_{1}^{*}+\omega_{2}+\omega_{e 0}^{*}$ and $\tau-\omega_{2}=i\left(e_{1} d z /(z-\zeta)+\Phi d e_{1}\right)^{*}+$ $e_{2} \varphi+\omega_{1}^{*}+\omega_{e 0}^{*}$. The left hand side has the same periods about $\delta$ as $\theta$, and so does the right hand side. It follows that $\tilde{\tau}=\tau-\omega_{2}$ and $\tilde{\tau}^{*}$ have the same periods about $\delta$ as the given $\theta$. (They have actually on $\underline{W}$ the same periods as $\Theta$ ).

In particular, if there exists on $\underline{W}$ a differential $\phi^{\prime}$ analytic with zero period along $\delta$, we can repeat the construction outlined in $\S 3 \mathrm{~A}$ and get differentials $\tilde{\tau}$ and $\tilde{\tau}^{*}$ with zero periods about $\delta$.

3C. We may write the decomposition

$$
\tilde{\tau}=\tilde{\psi}+\overline{\tilde{\chi}}
$$

where $\chi$ is analytic and $\psi$ is analytic except for the singularity at $z=\zeta$. If $\tilde{\tau}$ and $\tilde{\tau}^{*}$ have zero period about $\delta$, the same is true for $\tilde{\psi}$ and $\tilde{\chi}$ for:

$$
\begin{aligned}
& \tilde{\psi}=\frac{1}{2}\left(\tilde{\tau}+i \tau^{*}\right) \\
& \tilde{\chi}=\frac{1}{2}\left(\tilde{\tau}-i \tau^{*}\right) .
\end{aligned}
$$

Notice that $\tilde{\tau}=\tau$ for $\gamma=\beta$.

3D. Let $\Delta$ be the disk $|z|<r_{1}$. On $\underline{W},(\varphi+\bar{\varphi}) / 2 \in \Gamma_{h e} \cap \Gamma_{h 0}$. We shall call $d g=\frac{1}{2}(\varphi+\bar{\varphi})$, where $g$ is harmonic and constant on every component of the boundary of $\underline{W}$. In $4, \frac{1}{2}[d z /(z-\zeta)+\bar{d} \bar{z} /(\bar{z}-\bar{\zeta})]$ is the differential of $\log |z-\zeta|$. To sum up we have here:

$$
(\Theta+\bar{\Theta}) / 2=d\left(e_{1} \log |z-\zeta|\right)+d\left(e_{2} g\right) .
$$


By the procedure outlined in $\S 3 \mathrm{~A}$ we obtain a differential $(\tau+\bar{\tau}) / 2$, which is harmonic exact. Putting $(\tau+\bar{\tau}) / 2=d h, h$ is constant on every component of $\beta(W)$.

3E. We show here that one may get a function $h$ which is constant along $\beta$. Let $\sigma\left(\alpha_{0}\right)$ be defined as in $\S 3 \mathrm{~A} . \sigma\left(\alpha_{0}\right)^{*} \in \Gamma_{h 0}^{*}(W)$, therefore $\sigma\left(\alpha_{0}\right)^{*} \notin \Gamma_{h e}(\underline{W})$. Then $\sigma\left(\alpha_{0}\right)^{*}$ has a nonzero period along $\alpha_{0}$ and $\sigma\left(\alpha_{0}\right)^{*} \notin \Gamma_{h\left(\text { sed }_{0}\right)}(W)$. It follows that $\sigma\left(\alpha_{0}\right) \notin \Gamma_{h\left(\text { seo }_{0}\right)}^{*}$ and the orthogonal projection of $\sigma\left(\alpha_{0}\right)$ on $\Gamma_{h e(0 \beta)} \cap \Gamma_{h\left(0 \alpha_{0}\right)}$ is not zero. (Theorem 2C.) Let $\sigma^{\prime}\left(\alpha_{0}\right)$ be that projection; using $\sigma^{\prime}\left(\alpha_{0}\right)$ instead of $\sigma\left(\alpha_{0}\right)$ in the previous construction one gets a function $h$ with the required property, say $h_{0}$. We suggest for $d h_{0}$ the name of Green's differential, and for the corresponding $\tau$, say $\tau_{0}$, the name of capacity differential.

$3 \mathrm{~F}$. Let us now consider a closed partition of $\beta$ into $\gamma$ and $\delta$; put $\alpha_{0} \cup \gamma=\gamma^{\prime}$. We consider here instead of $\sigma^{*}\left(\alpha_{0}\right)$ the projection of $\sigma^{*}\left(\alpha_{0}\right)$ on $\Gamma_{h(s e \delta)}$. This is equivalent to subtracting from $\sigma^{*}\left(\alpha_{0}\right)$ a quantity which is an element of $\Gamma_{h e(0 \delta)}^{*} \cap \Gamma_{h\left(0 \gamma^{\prime}\right)}^{*}$ : (This means that the remaining part of $\sigma\left(\alpha_{0}\right)$ is still an element of $\Gamma_{h e} \cap \Gamma_{h 0}$.) We get a nonzero projection if and only if $\sigma\left(\alpha_{0}\right) \notin \Gamma_{h(0 \gamma)} \cap \Gamma_{h e\left(0 \gamma^{\prime}\right)}$ i.e. putting $\sigma\left(\alpha_{0}\right)=d f, f$ should have different constant values on $\alpha_{0}$ and $\gamma$. We shall call the differential $\tau$ thus obtained a capacity differential for the boundary part $\gamma$. If $\gamma$ is a component of $\beta$, we get the capacity differential of the boundary component $\gamma$.

\section{Reproducing properties.}

4A. We shall assume first that $W$ is the interior of a compact bordered surface. Let us call $\alpha$ the circle $|z-\zeta|=r$ and set $W_{0}=$ $W-\{|z-\zeta|<r\}$. Let $\tau_{0}$ be Green's differential, and $\Theta_{0}$ the corresponding singularity. For $\omega=d f \in \Gamma_{h e}$ we write down the generalized Green's formula on $W_{0}$ :

$$
\left(\omega,\left(\tau_{0}+\bar{\tau}_{0}\right) / 2\right)-\left(\omega^{*},\left(\tau_{0}+\bar{\tau}_{0}\right)^{*} / 2\right)=0 .
$$

or

$$
\int_{\beta-\alpha} f\left(\tau_{0}+\bar{\tau}_{0}\right)^{*} / 2-h_{0} d f^{*}=0 \text {. }
$$

First, $h_{0}$ being 0 on $\beta, \int_{\beta} h_{0} d f^{*}=0$. Therefore:

$$
\int_{\beta} f\left(\tau_{0}+\bar{\tau}_{0}\right)^{*} / 2=\int_{\alpha} f\left(\tau_{0}+\bar{\tau}_{0}\right)^{*} / 2-h_{0} d f^{*} .
$$

Let now $W_{0} \rightarrow W$, or $r \rightarrow 0$. For $r=\varepsilon$ on $|z|=r, h_{0}=\log |z-\zeta|+r_{11}(z)$. 
where $\eta_{1}(z)$ is bounded. It follows that $\lim _{r \rightarrow 0} \int_{a} h_{0} d f^{*}=0$. Now on $|z|<r$,

$$
\frac{1}{2}\left(\tau_{0}+\bar{\tau}_{0}\right)^{*}=(\Theta+\bar{\Theta} / 2)^{*}+\eta_{2}(z),
$$

where $\eta_{2}(z)$ is bounded. Moreover:

$$
(\Theta+\bar{\Theta})^{*} / 2=(-i \Theta+i \bar{\Theta}) / 2=-i(\Theta-\bar{\Theta}) / 2=d \arg (z-\zeta) .
$$

Therefore:

$$
\lim _{r \rightarrow 0} \int_{\alpha} f\left(\tau_{0}+\bar{\tau}_{0}\right)^{*} / 2=\lim _{r \rightarrow 0} \int_{\alpha} f d \arg (z-\zeta)=2 \pi f(\zeta) .
$$

We now may state the following theorem:

THEOREM. For all harmonic functions $f$ or $W$, the differential $\tau_{0}+\bar{\tau}_{0} / 2$ has the following reproducing property:

$$
\int_{\beta} f\left(\tau_{0}+\bar{\tau}_{0}\right)^{*} / 2=2 \pi f(\zeta)
$$

4B. If we now use $h$ instead of $h_{0}$ we need to restrict $d f$ to the class $\Gamma_{h e} \cap \Gamma_{h s e}^{*}$ and state:

THEOREM. For all harmonic functions $f$ on $W$ whose conjugate periods vanish along all dividing cycles, the differential $\tau+\bar{\tau} / 2$ satisfies:

$$
\int_{\beta} f(\tau+\bar{\tau}) / 2=2 \pi f(\zeta)
$$

4C. Green's differential enjoys another important property:

TheOREM. Let $d f \in \Gamma_{h e}$, and $\tau_{0}$ be Green's differential. Then:

$$
\left(d f,\left(\tau_{0}+\bar{\tau}_{0}\right)^{*} / 2\right)=0 \text {. }
$$

Proof. $\quad\left(d f,\left(\tau_{0}+\bar{\tau}_{0}\right)^{*} / 2\right)=\left(d f,\left(\Theta_{0}+\bar{\Theta}_{0}\right)^{*} / 2\right)$

$$
=-\lim _{r \rightarrow 0} \int_{\beta-\alpha} f\left(\Theta_{0}+\bar{\Theta}_{0}\right) / 2=\lim _{r \rightarrow 0} \int_{\alpha} f\left(\Theta_{0}+\bar{\Theta}_{0}\right) / 2 .
$$

4D. We shall now extend Theorem $4 \mathrm{C}$ to open Riemann surfaces. Let $W$ be an open Riemann surface and $\{\Omega\}$ a canonical exhaustion. Let $d F_{\Omega}=\left(\varphi_{0, \Omega}+\bar{\varphi}_{0 \Omega \Omega}\right) / 2$; we know that $d F_{\Omega} \in \Gamma_{h e(0 \beta)} \cap \Gamma_{h(0 \alpha)}$ on $\Omega-\delta$. If $d F=\left(\varphi_{0}+\bar{\varphi}_{0}\right) / 2$, we obtain easily by a reasoning analogous to the one in (I, Chapter V. $\S 14$. C) that

$$
\lim _{\Omega \rightarrow W}\left\|d F-d F_{\Omega}\right\|_{\Omega-\delta}=0 \text {. }
$$


We recall that $(\Theta+\bar{\Theta}) / 2=d\left(e_{1} \log |z-\zeta|\right)+d\left(e_{2} F\right)$. We now have:

$$
\begin{aligned}
(d f, & \left.\left(\tau_{0}+\bar{\tau}_{0}\right)^{*} / 2\right)=\left(d f,\left(\Theta_{0}+\bar{\Theta}_{0}\right)^{*} / 2\right) \\
& =\lim _{\Omega \rightarrow W}\left(d f,\left(\Theta_{0}+\bar{\Theta}_{0}\right)^{*} / 2\right)_{\Omega} \\
& =\lim _{\Omega \rightarrow W}\left(d f, \frac{1}{2}\left(\Theta_{0}+\bar{\Theta}_{0}\right)^{*}-\frac{1}{2}\left(\Theta_{0 \Omega}+\bar{\Theta}_{0 \Omega}\right)^{*}\right)_{\Omega} \\
& =\lim _{\Omega \rightarrow W}\left(d f, d\left(e_{2} F\right)^{*}-d\left(e_{2} F\right)^{*}\right)_{\Omega} \\
& =\lim _{\Omega \rightarrow W}\left(d f, d\left(e_{2} F\right)^{*}-d\left(e_{2} F_{\Omega}\right)^{*}\right)_{\Omega-\delta} .
\end{aligned}
$$

Now let $A$ be the compact set $\left\{z: r_{1} \leqq|z| \leqq r_{2}\right\}$ and let $\Omega-\delta=A \cup A^{\prime}$. We have:

$$
\begin{aligned}
& \left\|d\left(e_{2} F\right)^{*}-d\left(e_{2} F_{\Omega}\right)^{*}\right\|_{\Omega-\delta} \\
& \quad=\left\|d\left(e_{2} F\right)-d\left(e_{2} F_{\Omega}\right)\right\|_{\Omega-\delta} \\
& \quad=\left\|d e_{2}\left(F-F_{\Omega}\right)\right\|_{A}+\left\|d F-d F_{\Omega}\right\|_{A^{\prime}} .
\end{aligned}
$$

Because $\left\|d F-d F_{\Omega}\right\|_{A} \rightarrow 0$ as $\Omega \rightarrow W, F \rightarrow F_{\Omega}$ uniformly on $A$ hence $\lim _{\Omega \rightarrow W}\left\|d e_{2}\left(F-F_{\Omega}\right)\right\|_{A}=0$. Now on $A^{\prime}$

$$
\lim _{\Omega \rightarrow W}\left\|d F-d F_{\Omega}\right\|_{A^{\prime}} \leqq \lim _{\Omega \rightarrow W}\left\|d F-d F_{\Omega}\right\|_{\Omega-\delta}=0 .
$$

It follows that $\lim _{\Omega \rightarrow W}|| d\left(e_{2} F\right)^{*}-d\left(e_{2} F_{\Omega}\right)^{*} \|_{\Omega-\delta}=0$ and $\left|\left(d f,\left(\tau_{0}+\bar{\tau}_{0}\right) / 2\right)\right| \leqq$ $\lim _{\Omega \rightarrow W}\|d f\|_{\Omega-\delta}\left\|d\left(e_{2} F\right)^{*}-d\left(e_{2} F_{\Omega}\right)^{*}\right\|_{\Omega-\delta}=0$, which proves the theorem.

\section{BIBLIOGRAPHY}

1. L. V. Ahlfors \& L. Sario, Riemann surfaces, Princeton University Press: Princeton, 1960.

UNIVERSity OF CALIFornia, Los ANGEleS 


\title{
PACIFIC JOURNAL OF MATHEMATICS
}

\author{
EDITORS
}

Ralph S. Phillips

Stanford University

Stanford, California

M. G. Arsove

University of Washington

Seattle 5, Washington
A. L. Whiteman

University of Southern California Los Angeles 7, California

Lowell J. Paige

University of California

Los Angeles 24, California

\section{ASSOCIATE EDITORS}
E. F. BECKENBACH
D. DERRY
M. OHTSUKA
H. L. ROYDEN
E. SPANIER
E. G. STRAUS
T. M. CHERRY
F. WOLF

\section{SUPPORTING INSTITUTIONS}

\author{
UNIVERSITY OF BRITISH COLUMBIA \\ CALIFORNIA INSTITUTE OF TECHNOLOGY \\ UNIVERSITY OF CALIFORNIA \\ MONTANA STATE UNIVERSITY \\ UNIVERSITY OF NEVADA \\ NEW MEXICO STATE UNIVERSITY \\ OREGON STATE UNIVERSITY \\ UNIVERSITY OF OREGON \\ OSAKA UNIVERSITY \\ UNIVERSITY OF SOUTHERN CALIFORNIA
}

\author{
STANFORD UNIVERSITY \\ UNIVERSITY OF TOKYO \\ UNIVERSITY OF UTAH \\ WASHINGTON STATE UNIVERSITY \\ UNIVERSITY OF WASHINGTON \\ $*$
AMERICAN MATHEMATICAL SOCIETY \\ CALIFORNIA RESEARCH CORPORATION \\ SPACE TECHNOLOGY LABORATORIES \\ NAVAL ORDNANCE TEST STATION
}

Mathematical papers intended for publication in the Pacific Journal of Mathematics should be typewritten (double spaced), and the author should keep a complete copy. Manuscripts may be sent to any one of the four editors. All other communications to the editors should be addressed to the managing editor, L. J. Paige at the University of California, Los Angeles 24, California.

50 reprints per author of each article are furnished free of charge; additional copies may be obtained at cost in multiples of 50 .

The Pacific Journal of Mathematics is published quarterly, in March, June, September, and December. Effective with Volume 13 the price per volume (4 numbers) is $\$ 18.00$; single issues, $\$ 5.00$. Special price for current issues to individual faculty members of supporting institutions and to individual members of the American Mathematical Society: $\$ 8.00$ per volume; single issues $\$ 2.50$. Back numbers are available.

Subscriptions, orders for back numbers, and changes of address should be sent to Pacific Journal of Mathematics, 103 Highland Boulevard, Berkeley 8, California.

Printed at Kokusai Bunken Insatsusha (International Academic Printing Co., Ltd.), No. 6, 2-chome, Fujimi-cho, Chiyoda-ku, Tokyo, Japan.

PUBLISHED BY PACIFIC JOURNAL OF MATHEMATICS, A NON-PROFIT CORPORATION

The Supporting Institutions listed above contribute to the cost of publication of this Journal, but they are not owners or publishers and have no responsibility for its content or policies. 


\section{Pacific Journal of Mathematics}

\section{Vol. 12, No. $2 \quad$ February, 1962}

William George Bade and Robert S. Freeman, Closed extensions of the Laplace operator determined by a general class of boundary conditions . . . . . . . 395

William Browder and Edwin Spanier, H-spaces and duality ............. 411

Stewart S. Cairns, On permutations induced by linear value functions . . . . . . . 415

Frank Sydney Cater, On Hilbert space operators and operator roots of

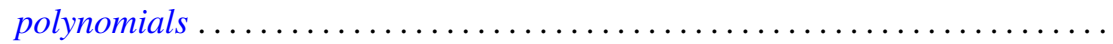

Stephen Urban Chase, Torsion-free modules over $K[x, y] \ldots \ldots \ldots \ldots \ldots \ldots \ldots 437$

Heron S. Collins, Remarks on affine semigroups . . . . . . . . . . . . . . . . 449

Peter Crawley, Direct decompositions with finite dimensional factors . . . . . . . 457

Richard Brian Darst, A continuity property for vector valued measurable

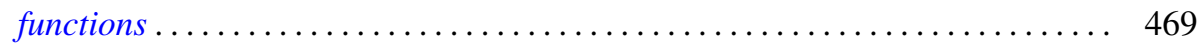

R. P. Dilworth, Abstract commutative ideal theory ................. 481

P. H. Doyle, III and John Gilbert Hocking, Continuously invertible spaces . . . . . . 499

Shaul Foguel, Markov processes with stationary measure . . . . . . . . . . . 505

Andrew Mattei Gleason, The abstract theorem of Cauchy-Weil ............ 511

Allan Brasted Gray, Jr., Normal subgroups of monomial groups . . . . . . . . . . 527

Melvin Henriksen and John Rolfe Isbell, Lattice-ordered rings and function

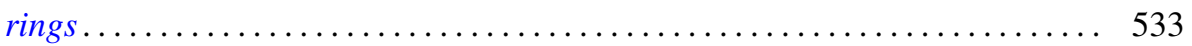

Amnon Jakimovski, Tauberian constants for the $[J, f(x)]$ transformations . ..... 567

Hubert Collings Kennedy, Group membership in semigroups . . . . . . . . . . . 577

Eleanor Killam, The spectrum and the radical in locally $m$-convex algebras ..... 581

Arthur H. Kruse, Completion of mathematical systems . . . . . . . . . . . . . 589

Magnus Lindberg, On two Tauberian remainder theorems ................ 607

Lionello A. Lombardi, A general solution of Tonelli's problem of the calculus of

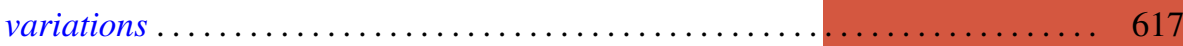

Marvin David Marcus and Morris Newman, The sum of the elements of the powers

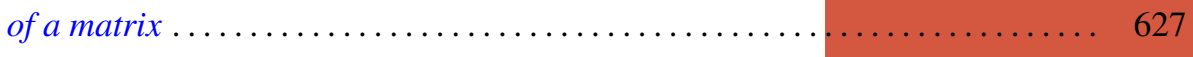

Michael Bahir Maschler, Derivatives of the harmonic measures in

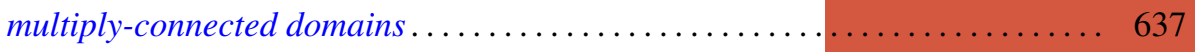

Deane Montgomery and Hans Samelson, On the action of $\mathrm{SO}(3)$ on $S^{n} \ldots \ldots \ldots 649$

J. Barros-Neto, Analytic composition kernels on Lie groups . . . . . . . . . . . . 661

Mario Petrich, Semicharacters of the Cartesian product of two semigroups ...... 679

John Sydney Pym, Idempotent measures on semigroups . . . . . . . . . . . . 685

K. Rogers and Ernst Gabor Straus, A special class of matrices . . . . . . . . . . . . 699

U. Shukla, On the projective cover of a module and related results . . . . . . . . . 709

Don Harrell Tucker, An existence theorem for a Goursat problem . . . . . . . . . . . 719

George Gustave Weill, Reproducing kernels and orthogonal kernels for analytic

differentials on Riemann surfaces ......................... 729

George Gustave Weill, Capacity differentials on open Riemann surfaces ........ 769

G. K. White, Iterations of generalized Euler functions . . . . . . . . . . . . . 777

Adil Mohamed Yaqub, On certain finite rings and ring-logics . . . . . . . . . 785 